\title{
Electronic structure and coexistence of superconductivity with magnetism in $\mathrm{RbEuFe}_{4} \mathrm{As}_{4}$
}

\author{
T. K. Kim $\odot,{ }^{1, *}$ K. S. Pervakov $\odot,{ }^{2}$ D. V. Evtushinsky $\odot,{ }^{3}$ S. W. Jung $\odot,{ }^{1,4}$ G. Poelchen $\odot,{ }^{5,6}$ K. Kummer $\odot,{ }^{6}$ V. A. Vlasenko $\odot,{ }^{2}$ \\ A. V. Sadakov $\odot,{ }^{2}$ A. S. Usoltsev, ${ }^{2}$ V. M. Pudalov $\odot,{ }^{2}$ D. Roditchev, ${ }^{7,8,9}$ V. S. Stolyarov $\odot,{ }^{9,10}$ D. V. Vyalikh $\odot,{ }^{11,12}$ \\ V. Borisov, ${ }^{13}$ R. Valentí, ${ }^{13}$ A. Ernst, ${ }^{14,15}$ S. V. Eremeev $\odot,{ }^{16,17}$ and E. V. Chulkov ${ }^{11,17,18}$ \\ ${ }^{1}$ Diamond Light Source, Harwell Campus, Didcot, OX11 ODE, United Kingdom \\ ${ }^{2}$ Ginzburg Center for High Temperature Superconductivity and Quantum Materials Lebedev Physical Institute, Moscow 119991, Russia \\ ${ }^{3}$ Laboratory for Quantum Magnetism, Institute of Physics, École Polytechnique Fédérale de Lausanne, CH-1015 Lausanne, Switzerland \\ ${ }^{4}$ Department of Physics and Research Institute of Natural Science, Gyeongsang National University, Jinju 52828, Republic of Korea \\ ${ }^{5}$ Institut für Festkörper- und Materialphysik, Technische Universität Dresden, D-01062, Dresden, Germany \\ ${ }^{6}$ European Synchrotron Radiation Facility, 71 Avenue des Martyrs, 38043 Grenoble, France \\ ${ }^{7}$ LPEM, ESPCI Paris, PSL Research University, CNRS, 75005 Paris, France \\ ${ }^{8}$ Sorbonne Universite, CNRS, LPEM, 75005, Paris, France \\ ${ }^{9}$ Moscow Institute of Physics and Technology, 141700 Dolgoprudny, Russia \\ ${ }^{10}$ N. L. Dukhov All-Russia Research Institute of Automatics, 127055 Moscow, Russia \\ ${ }^{11}$ Donostia International Physics Center (DIPC), 20018 Donostia-San Sebastián, Basque Country, Spain \\ ${ }^{12}$ IKERBASQUE, Basque Foundation for Science, 48013 Bilbao, Spain \\ ${ }^{13}$ Institut für Theoretische Physik, Goethe-Universität Frankfurt, Max-von-Laue-Strasse 1, D-60438 Frankfurt am Main, Germany \\ ${ }^{14}$ Institut für Theoretische Physik, Johannes Kepler Universität, A 4040 Linz, Austria \\ ${ }^{15}$ Max-Planck-Institut für Mikrostrukturphysik, Weinberg 2, D-06120 Halle, Germany \\ ${ }^{16}$ Institute of Strength Physics and Materials Science, Russian Academy of Sciences, 634055 Tomsk, Russia \\ ${ }^{17}$ Saint Petersburg State University, 198504 Saint Petersburg, Russia \\ ${ }^{18}$ Departamento de Polímeros y Materiales Avanzados: Física, Química y Tecnología, Facultad de Ciencias Químicas, Universidad del País \\ Vasco UPV/EHU, 20080 San Sebastián/Donostia, Basque Country, Spain
}

(Received 13 July 2020; revised 27 March 2021; accepted 4 May 2021; published 25 May 2021)

\begin{abstract}
In the novel stoichiometric iron-based material $\mathrm{RbEuFe}_{4} \mathrm{As}_{4}$, superconductivity coexists with a peculiar longrange magnetic order of Eu $4 \mathrm{f}$ states. Using angle-resolved photoemission spectroscopy, we reveal a complex three-dimensional electronic structure and compare it with density functional theory calculations. Multiple superconducting gaps were measured on various sheets of the Fermi surface. High-resolution resonant photoemission spectroscopy reveals magnetic order of the Eu $4 \mathrm{f}$ states deep into the superconducting phase. Both the absolute values and the anisotropy of the superconducting gaps are remarkably similar to the sibling compound without $\mathrm{Eu}$, indicating that Eu magnetism does not affect the pairing of electrons. A complete decoupling between $\mathrm{Fe}$ and Eu-derived states was established from their evolution with temperature, thus unambiguously demonstrating that superconducting and a long-range magnetic orders exist independently from each other. The established electronic structure of $\mathrm{RbEuFe}_{4} \mathrm{As}_{4}$ opens opportunities for the future studies of the highly unorthodox electron pairing and phase competition in this family of iron-based superconductors with doping.
\end{abstract}

DOI: 10.1103/PhysRevB.103.174517

\section{INTRODUCTION}

Even after many years of intensive research, the mechanism of the electron pairing in iron-based superconductors (IBSC) is still not well understood. It is known that the emergence of superconductivity (SC) with doping is accompanied by a suppression of the structural and magnetic transitions observed in undoped parent compounds. Theoretical considerations suggest that the pairing in IBSCs might be due to

\footnotetext{
*Corresponding author: timur.kim@diamond.ac.uk
}

Published by the American Physical Society under the terms of the Creative Commons Attribution 4.0 International license. Further distribution of this work must maintain attribution to the author(s) and the published article's title, journal citation, and DOI. the spin-fluctuation exchange [1-4]. In the case of weakly doped compounds, the superconducting gap should have a $s^{ \pm}$symmetry. However, numerous studies of these materials demonstrate that the physics of the pairing is more complex because of the multiorbital and multiband nature of lowenergy fermionic excitations [5-7]. It turns out that both the symmetry and the structure of the order parameter result from a rather nontrivial interplay between spin-fluctuation exchange, intra-band Coulomb repulsion, and the momentum structure of the competing interactions $[8,9]$.

In a newly discovered class of IBSCs, the so-called 1144 family [10], there are particularly interesting examples of $A \mathrm{EuFe}_{4} \mathrm{As}_{4}(A=\mathrm{Rb}, \mathrm{Cs})$ compounds where SC coexists with unusual Eu-magnetic order [11-14]. The crystal structure of these compounds [see Fig. 1(a)] can be viewed as an intergrowth between undoped $\mathrm{EuFe}_{2} \mathrm{As}_{2}$ and 
(a)
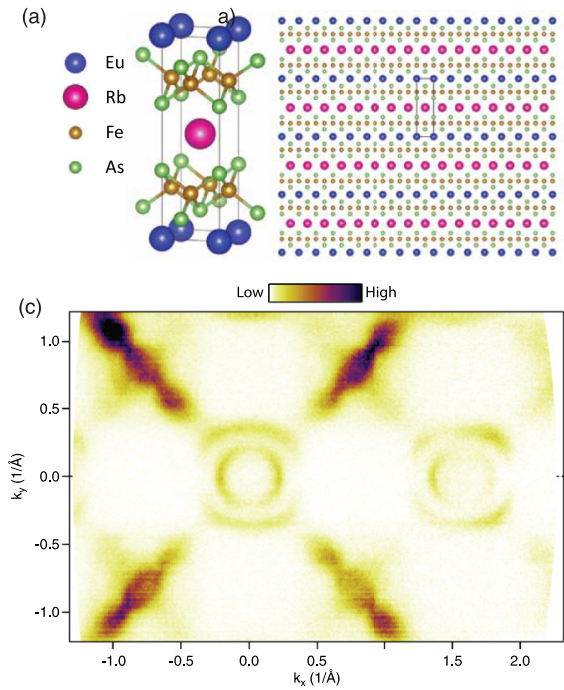

(e)

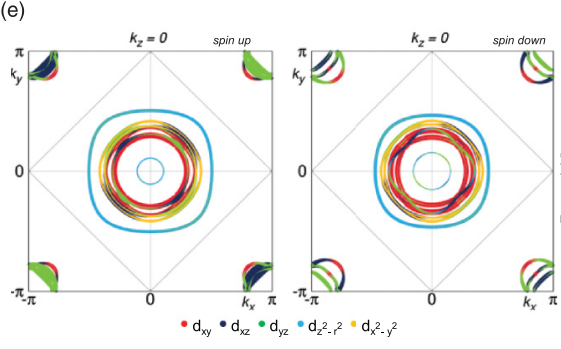

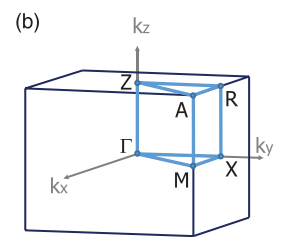
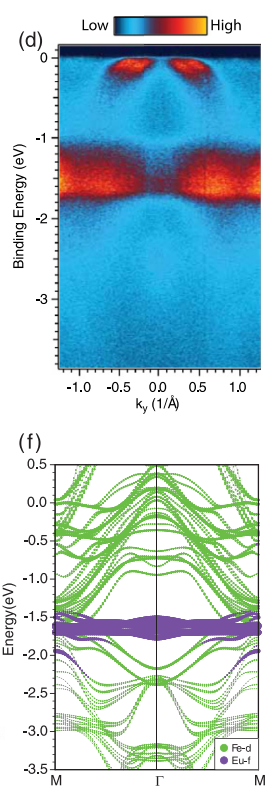

FIG. 1. Structure and FS topology. (a) Layered crystal structure and (b) bulk Brillouin zone of $\mathrm{RbEuFe}_{4} \mathrm{As}_{4}$. (c) The $k_{x}-k_{y}$ FS map, measured using $70 \mathrm{eV}$ photons in linear vertical (LV) polarization. (d) Band dispersions in $\mathrm{M}-\Gamma-\mathrm{M}$ direction measured using $113 \mathrm{eV}$ photons for LV polarization. All data measured at $40 \mathrm{~K}$. (e) Calculated FS for $k_{z}=0$ for FM configuration of Eu moments. (f) Calculated band dispersions in $\mathrm{M}-\Gamma-\mathrm{M}$ direction showing $\mathrm{Fe} 3 \mathrm{~d}$ (green) and $\mathrm{Eu} 4 \mathrm{f}$ (blue) bands.

heavily overdoped $A \mathrm{Fe}_{2} \mathrm{As}_{2}$. As a result, $\mathrm{RbEuFe}_{4} \mathrm{As}_{4}$ becomes intrinsically hole-doped, exhibiting SC with a high transition temperature $\sim 36 \mathrm{~K}$. Moreover, the evidence of in-plane ferromagnetic (FM) ordering of the $\mathrm{Eu}^{2+}$ spins below $\sim 15 \mathrm{~K}$ was given by magnetization measurements for both polycrystalline [15] and single crystal samples [16,17]. The optical conductivity measurements [18] revealed a fully opened SC gap of about $5 \mathrm{meV}$, while the inelastic neutron scattering measurements showed spin resonance at $18 \mathrm{meV}$, and a three-dimensional helical antiferromagnetic (AFM) order of the $\mathrm{Eu}$ atoms was determined from the neutron diffraction [19]. It has even been proposed that this exotic helical magnetic structure is generated by SC due to the interplay of Ruderman-Kittel-Kasuya-Yosida exchange interaction and macroscopic electromagnetic interaction between the superconducting and magnetic subsystems [20,21]. However, magnetic susceptibility and resistivity studies of $\mathrm{RbEuFe}_{4} \mathrm{As}_{4}$ under pressure found that the SC onset is suppressed monotonically by pressure while the magnetic transition was enhanced at higher pressures [13]. Muon spin rotation $(\mu \mathrm{SR})$ measurements of polycrystalline samples [22] indicated that under hydrostatic pressure the superconducting transition temperature $T_{c}$ decreased, while the magnetic

transition temperature $T_{m}$ increased, suggesting no coupling between $s^{ \pm}$SC typical for IBSC and the exotic magnetic order of Eu. A recent study of the magnetism and SC in Ni-doped $\mathrm{RbEuFe}_{4} \mathrm{As}_{4}$ showed that with doping the SC transition critical temperature $T_{c}$ decreased, but the Eu magnetic transition temperature $T_{m}$ is hardly affected [23]. This suggests that Eu magnetism and SC might be weakly dependent from each other.

In the present study, we elucidate the interdependence of $\mathrm{Fe}-\mathrm{SC}$ and Eu-magnetism in this novel class of materials by combining experimental observations with theoretical calculations. In particular, we investigate the question whether the spatial proximity of the ferromagnetically ordered layer of $\mathrm{Eu}$ atoms can induce a nontrivial pairing of the Fe conduction electrons with exotic symmetry of the superconducting gap.

\section{METHODS}

High quality single crystals of $\mathrm{RbEuFe}_{4} \mathrm{As}_{4}$ were grown by the self-flux technique. Superconducting and magnetic transition temperatures $\mathrm{T}_{\mathrm{c}} \sim 35 \mathrm{~K}$ and $\mathrm{T}_{\mathrm{m}} \sim 14 \mathrm{~K}$ were confirmed by magnetization measurements [24]. High-resolution angle-resolved photoemission spectroscopy (ARPES) and resonant photoemission spectroscopy (ResPES) measurements were performed at the I05 beamline at the Diamond Light Source, UK [25]. The photoelectron energy and angular distributions were analyzed with a SCIENTA R4000 hemispherical analyzer. The angular resolution was $0.2^{\circ}$, and the overall energy resolution was better than $10 \mathrm{meV}$ for Fermi surface (FS) mapping and $\sim 2-3 \mathrm{meV}$ for SC gap measurements. Samples were top-post cleaved in situ at pressures better than $2 \times 10^{-10}$ mbar at temperatures under $10 \mathrm{~K}$. In order to increase the contrast in two dimensional color plot of resonant photoemission signal we have used a procedure described in Ref. [26]. X-ray magnetic circular dichroism (XMCD) measurements were performed at the HECTOR end-station of the BOREAS beamline at the ALBA synchrotron radiation facility [27]. Absorption spectra in total electron yield mode have been recorded at the $\mathrm{Fe} \mathrm{L}_{2,3}$ and $\mathrm{Eu}_{4,5}$ edges in magnetic fields up to $6 \mathrm{~T}$ and at $5 \mathrm{~K}$ sample temperature, well below $\mathrm{T}_{\mathrm{m}} \sim 14 \mathrm{~K}$. For Andreev reflection spectroscopy we used the "break-junction" (BJ) technique [28,29] with crystals from the same batch. The samples were attached to a springy sample holder and supplied with four-contact pads enabling 4-point measurements of the current voltage $(I-V)$ and dynamic conductance $(d I / d V$ vs $V)$ characteristics. In order to generate a microcrack in the bulk crystal, the samples were mechanically bent in liquid helium environment at $4.2 \mathrm{~K}$, thus preserving the contamination- and oxide-free junction. To operate in a superconductor-normal metal-superconductor (S-N-S) regime, the microcrack was precisely mechanically tuned until we achieved the desired Andreev-mode $I-V$ characteristic seen as excess conductance in the vicinity of zero bias [30]. For more details on the BJ experimental technique, see Refs. [31,32].

Density functional theory (DFT) calculations were performed considering various basis sets [33]: the projector augmented-wave method for representation of core electrons $[34,35]$ as implemented in the VASP code [36], the all- 
electron full-potential localized orbitals (FPLO) basis set code [37], and a full potential Green's function method within the multiple scattering theory [38]. Calculations were benchmarked with various codes. The generalized gradient approximation (GGA-PBE) [39] to the exchange-correlation potential was applied. To perform the calculations we have used the experimental crystal structure parameters [15]. The $\mathrm{Eu} 4 \mathrm{f}$ states were treated employing the GGA+U approach [40] within the Dudarev scheme [41]. The $\mathrm{U}_{\text {eff }}=\mathrm{U}-\mathrm{J}$ value (where $\mathrm{U}$ and $\mathrm{J}$ are the effective on-site Coulomb and exchange interaction parameters, respectively) for the $\mathrm{Eu} 4 \mathrm{f}$ states was chosen to be equal to $5.5 \mathrm{eV}$. Using this $\mathrm{U}_{\text {eff }}$ value we found a good agreement with the experimentally obtained binding energy of the $\mathrm{Eu} 4 \mathrm{f}$ states of $\mathrm{RbEuFe}_{4} \mathrm{As}_{4}$ [42].

\section{RESULTS}

\section{A. Fermi surface topology}

In Fig. 1(c), we present a FS map obtained at $70 \mathrm{eV}$, corresponding to $k_{z}=0$ for the $\Gamma$ point where the hole pockets are largest. The measured FS is similar to optimally hole-doped $\mathrm{Ba}_{1-x} \mathrm{~K}_{x} \mathrm{Fe}_{2} \mathrm{As}_{2}$ (Ba “122") pnictides and consists of three holelike pockets at the center of the Brillouin zone $[\Gamma-\mathrm{Z}$ points in Fig. 1(b)] and propellerlike electronlike pockets at the corners of the the Brillouin zone [M-A points in Fig. 1(b)] [43]. STM study of the cold-cleaved surface of $\mathrm{RbEuFe}_{4} \mathrm{As}_{4}$ single crystals revealed the coexistence of large $\mathrm{Rb}$-terminated and small Eu-terminated terraces, both having $1 \times 2$ and $\sqrt{2} \times \sqrt{2}$ reconstructions [44]. For Ba"122" pnictides, the close proximity of the surface states, induced by such reconstructions, and bulk states can lead to the broadening of the ARPES spectra [45]. High symmetry direction band dispersions measured in $\mathrm{M}-\Gamma-\mathrm{M}$ direction in Fig. 1(d) show the $4 \mathrm{f}$ electron emission from bulk Eu atoms, between 1.0 and $1.7 \mathrm{eV}$ below the Fermi level. We note that there is no signature of $4 \mathrm{f}$ emission from surface Eu atoms, which would appear as an additional broad $4 \mathrm{f}$ signal at higher binding energies $[46,47]$. Apparently, the spectral pattern of Eu $4 \mathrm{f}$ states lies far away from the $\mathrm{Fe} 3 \mathrm{~d}$ states that are crossing the Fermi level. This observation also suggests that it is hard to anticipate any interaction between $\mathrm{Eu}$ and $\mathrm{Fe}$ sublattices [48].

The experimentally observed results are in good agreement with DFT calculations for $\mathrm{RbEuFe}_{4} \mathrm{As}_{4}$ [see Figs. 1(e) and 1(f)]. Like in many other known classes of IBSCs, DFT overestimates the size of both hole and electron FS, leading to the so-called "red-blue" shift of the Fe 3d bands: holelike bands at the center of the Brillouin zone move to higher binding energies, while electronlike bands at the corners of the Brillouin zone move to lower binding energies [49-51]. This results in smaller size FS observed in the experiment and in some cases to a corresponding increase of the density of states at the Fermi level due to the Van Hove singularity at the bottom of the electron pocket that moves toward zero energy at optimal doping [52]. This impedes direct quantitative comparison of the obtained DFT calculations with measured ARPES data. Such a task is beyond the scope of this study and will require the use of the Dynamical Mean Filed Theory or full Green function $(\mathrm{GW})$ methods to treat electron-electron correlations.
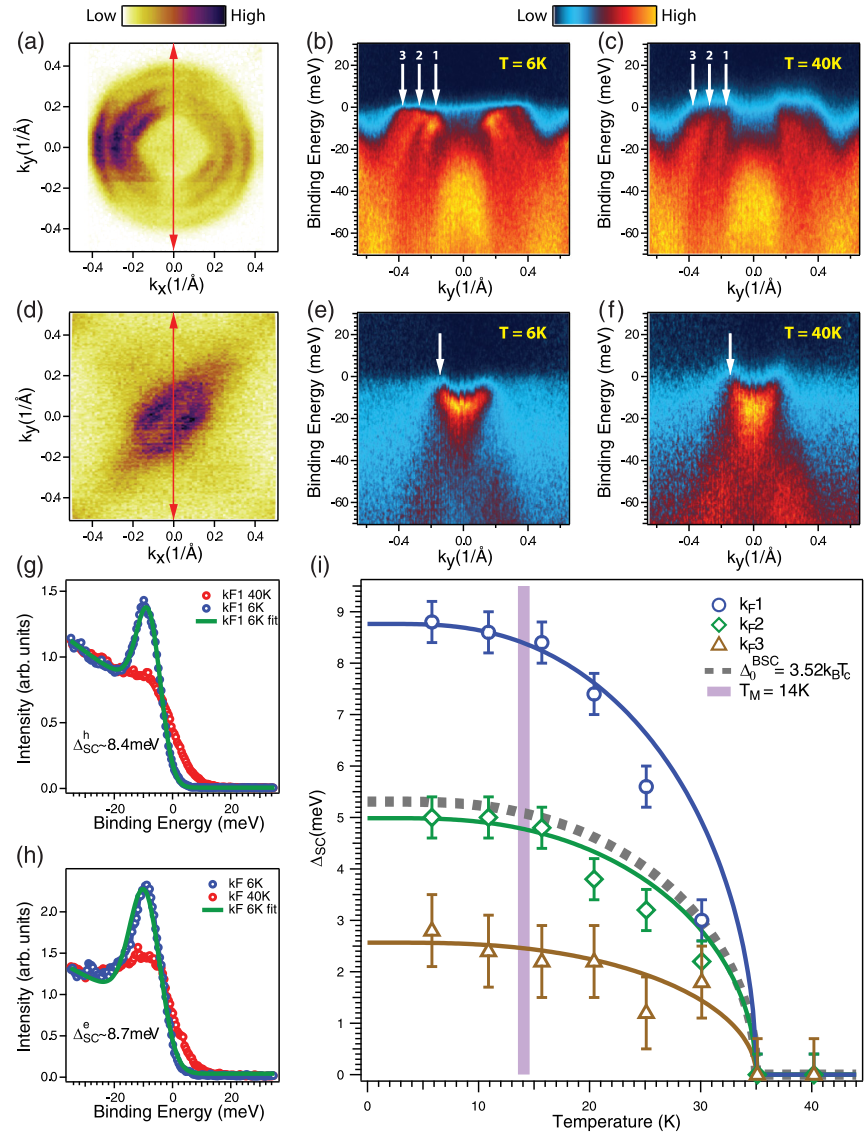

FIG. 2. Superconducting properties from ARPES. (a) Hole pocket FS at Z-point (40 K); (b, c) band dispersions at Z-point below and above $T_{c}$ measured along high symmetry direction are shown as a red double arrow in (a); white arrows indicate corresponding $k_{\mathrm{F}}$ positions; (d) Electron pocket FS at M-point $(40 \mathrm{~K})$; (e, f) band dispersions at $\mathrm{M}$-point below and above $\mathrm{T}_{\mathrm{c}}$ measured along high symmetry direction shown as a red double arrow in (d); white arrows indicate corresponding $k_{\mathrm{F}}$ positions; $(\mathrm{g}, \mathrm{h})$ photoemission spectra at equivalent $k_{\mathrm{F}}$ positions above and below $\mathrm{T}_{\mathrm{c}}$ together with the superconducting gap fit for hole and electron pockets; (i) temperature dependence of the superconducting gap obtained for three different FS sheets of the hole pocket at Z-point.

\section{B. Superconducting properties}

Using high-resolution ARPES, we have observed an opening of the superconducting gap on all sheets of the FS below $\mathrm{T}_{\mathrm{c}}$ (Fig. 2). The measured values of the gap at the hole pockets at the center of the Brillouin zone are significantly varying from one sheet to another, with the largest gap opening on the most inner holelike FS, similar to other Ba" 122 " pnictides [53-55]. We have also observed opening of the superconducting gap at the electron pocket. The obtained maximum value of the gap at the electron pocket is about $\sim 9 \mathrm{meV}$ according to the fit using the Dynes function [56] and is similar to the largest gap value for the hole pocket [Figs. 2(g) and 2(h)]. This is in a very good agreement with superconducting gap ARPES measurements for optimally doped $\mathrm{Ba}_{1-x} \mathrm{~K}_{x} \mathrm{Fe}_{2} \mathrm{As}_{2}$ [53-55]. These maximum gap values derived by ARPES are also in a very good agreement with published data from optical measurements of $\mathrm{RbEuFe}_{4} \mathrm{As}_{4}$ [18]. 

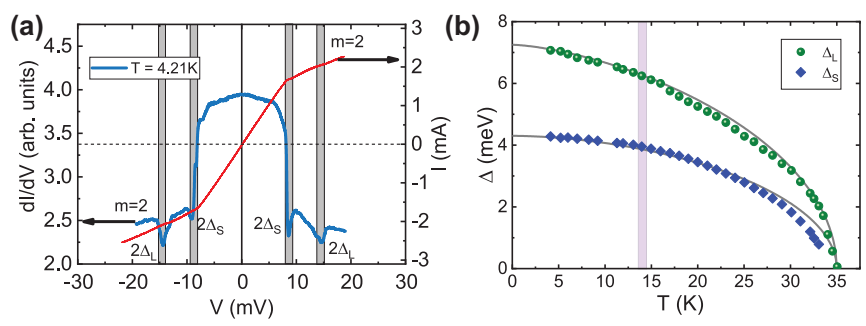

FIG. 3. Superconducting properties from Andreev reflection spectroscopy. (a) Typical $I-V$ (red) and dynamic conductance $d I / d V$ (blue) curves for double-stack junction $(\mathrm{m}=2)$. Gray strips highlight the dip positions for two gaps. (b) Temperature dependence of the two gaps derived from the voltage dips in dynamic conductance data of $m=2$ stack in the entire temperature range up to $T_{c}$. Green circles represent a larger gap and blue diamonds represent a smaller gap correspondingly. Gray lines show the BCS-like fit.

With increasing temperature, the superconducting gap gradually closes for all bands with classical BCS-type dependence, indicating a significant inter-orbital interaction of the Fe 3d bands [57-59], similar to sibling compound $\mathrm{CaKFe}_{4} \mathrm{As}_{4}$ [60] and Ba"122" family [55,61]. By measuring the temperature dependence of the superconducting gap at the hole pockets [Fig. 2(i)] within our energy resolution, we do not observe any change in the magnitude of the gap at the $\mathrm{Eu}$ magnetic transition $\mathrm{T}_{\mathrm{m}} \sim 14 \mathrm{~K}$.

In order to get deeper insight into the temperature evolution of the order parameter, we performed detailed measurements of the superconducting gap by Andreev reflection spectroscopy. The superconducting gap structure appears in the dynamic conductance ( $d I / d V$ vs $V)$ spectrum of S-N-S contact at temperatures $\mathrm{T}<\mathrm{T}_{\mathrm{c}}$ as a dip at certain bias voltage [28-30]. These dips are related with the first-order resonances at $V=2 \Delta_{\mathrm{i}} / e$, where $\Delta_{\mathrm{i}}$ is the gap value for a particular $i$-th $\mathrm{SC}$ condensate, and $e$ is the elementary charge. During sample cleaving in the BJ-experiments, beside the single S-N-S junction, stack contacts are also often formed with sequential chain of the several in-series connected S-N-S junctions. The stack contact resonances are observed at bias voltage $V_{m}=$ $m \times\left(2 \Delta_{i} / e\right)(m=2,3, \ldots)$ and may be easily disentangled by scaling down bias voltage with respect to integer $m$ [31]. For more details of Andreev reflection spectroscopy experiment, see Appendix B. On the dynamic conductance curve in Fig. 3(a), one can see dips at bias voltages $V= \pm 8.6$ and $\pm 14.5 \mathrm{meV}$, indicating the presence of two different gaps, the large $\Delta_{\mathrm{L}}$ and the small one $\Delta_{\mathrm{S}}$. With temperature rising, positions of the dips move to lower bias values and collapse to zero at the same $\mathrm{T}_{\mathrm{c}} \sim 35 \mathrm{~K}$, which coincides with the bulk value, determined from resistivity measurements. Tracing positions of the two major minima, we now plot the temperature dependencies of the two gaps in Fig. 3(b). By extrapolating the gap values to $T=0$ using BSC-like fit, we estimate $\Delta_{L}=$ $7.25 \pm 0.3 \mathrm{meV}, \Delta_{S}=4.3 \pm 0.2 \mathrm{meV}$, and the characteristic ratios $2 \Delta_{L} / k_{B} T_{c}=4.80,2 \Delta_{S} / k_{B} T_{c}=2.85$. We emphasize that both dependencies, $\Delta_{\mathrm{L}, \mathrm{S}}(T)$ pass smoothly and with no features through the temperature $\mathrm{T}_{\mathrm{m}} \sim 14 \mathrm{~K}$ of the Eu-AFM ordering, which is consistent with the momentum-resolved ARPES results [Fig. 2(i)]. Therefore, both our ARPES and Andreev reflection results suggest that the amplitude of the

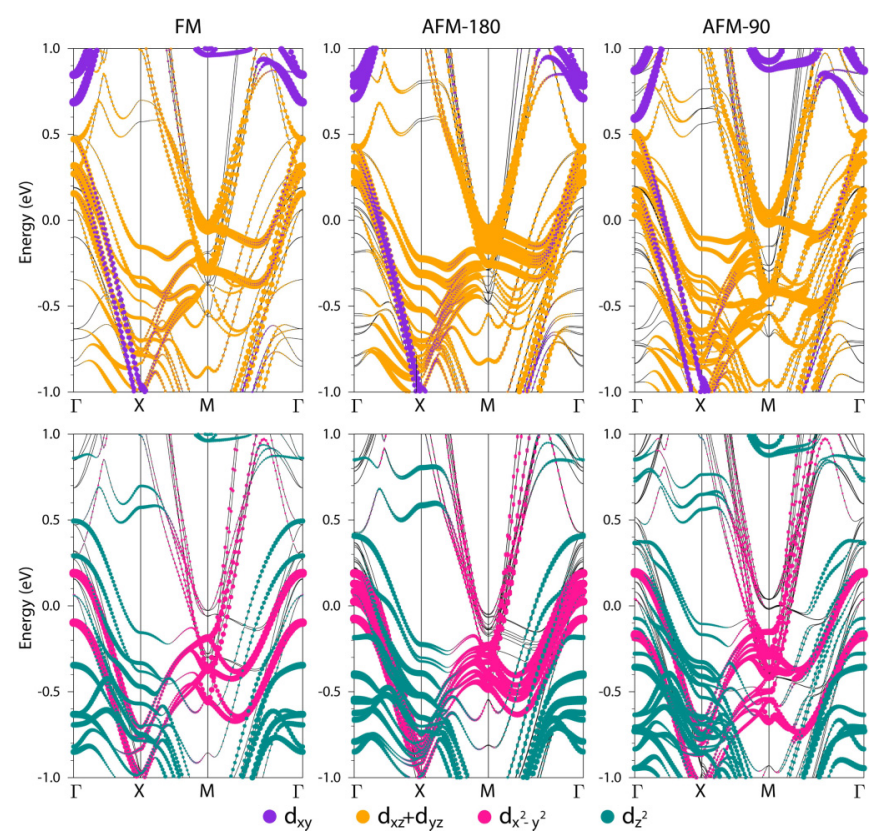

FIG. 4. DFT electronic structure. Band dispersions with the $\mathrm{Fe}$ 3d orbital character for FM (left column), A-type AFM (middle column), and helical AFM (right column) Eu magnetic structures. Calculations are done for 1,2 , and 4 formula units per cell correspondingly.

superconducting order parameter in $\mathrm{RbEuFe}_{4} \mathrm{As}_{4}$ might be fully insensitive to the magnetic ordering on the Eu sublattice.

In order to investigate the effects of Eu $4 \mathrm{f}$ magnetism on the $\mathrm{Fe} 3 \mathrm{~d}$ electronic states in the vicinity of the Fermi level, we performed band structure calculations within DFT for several types of Eu magnetic moment ordering: FM, A-type AFM ordering similar to $\mathrm{EuFe}_{2} \mathrm{As}_{2}$ (AFM180) [62,63], and for the recently proposed helical antiferromagnetic alignment of Eu moments (AFM90) [19]. As can be seen in Fig. 4, irrespective of magnetic ordering, the bands forming electron M-pockets at the Fermi level are almost completely determined by $\mathrm{d}_{x z(y z)} \mathrm{Fe}$ orbitals, whereas the bands forming hole $\Gamma$-pockets are contributed by a mixture of different $\mathrm{Fe} 3 \mathrm{~d}$ orbitals. In accordance with our calculations, the basic band structure at the Fermi level and the orbital character of Fe 3d bands are almost unaffected by different magnetic orderings of Eu $4 \mathrm{f}$ magnetic moments. Therefore, the underlying spinfluctuation-mediated interaction of $\mathrm{Fe} 3 \mathrm{~d}$ itinerant electrons responsible for superconducting pairing could be completely insensitive to the onset of the local magnetic order on Eu atoms.

\section{Magnetic properties}

In order to explore the magnetic properties of the Eu sublattice, we performed resonant photoemission measurements at the $\mathrm{Eu} 4 \mathrm{~d} \rightarrow$ 4f threshold using $142 \mathrm{eV}$ photons. This allows a resonant enhancement of the $4 \mathrm{f}$ emission from divalent $\mathrm{Eu}$ ions and gains insight exclusively into the spectral pattern of these states. The experiment was performed as a function of temperature going from the normal state $(40 \mathrm{~K})$ via the superconducting state $(20 \mathrm{~K})$ into the magnetic state $(7 \mathrm{~K})$ as shown 

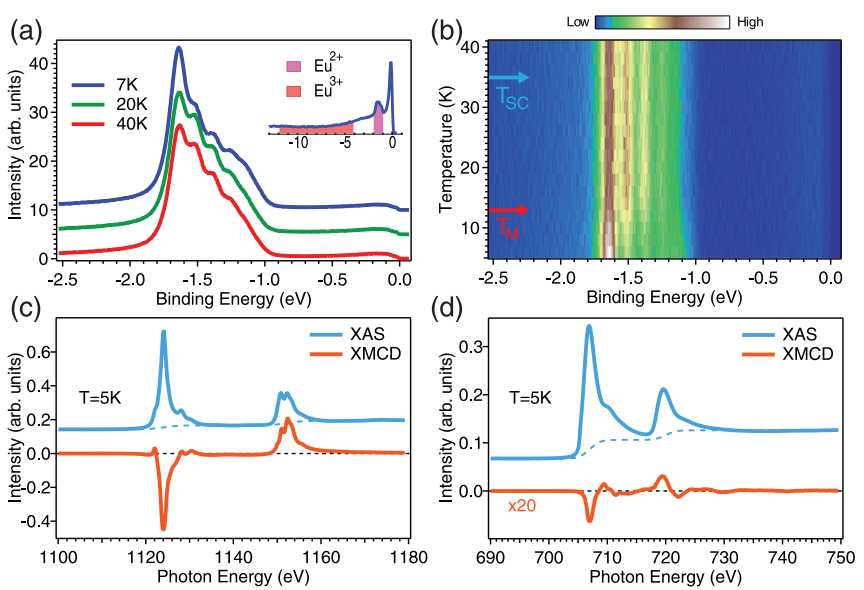

FIG. 5. Magnetic properties. (a) The resonant Eu $4 \mathrm{~d} \rightarrow 4 \mathrm{f}$ photoemission spectra taken at 7, 30, and $40 \mathrm{~K}$ with $142 \mathrm{eV}$ of photons and LV polarization; the off-resonant $120 \mathrm{eV}$ survey scan shown as an insert explicitly indicates no admixture of the trivalent Eu state. (b) Two-dimensional color plot presentation of the temperature evolution of the divalent Eu $4 \mathrm{f}$ resonant photoemission signal [64]. (c, d) XMCD spectra measured for $\mathrm{Eu}_{5,4}$ and $\mathrm{Fe} \mathrm{L}_{3,2}$ absorption edges.

in Figs. 5(a) and 5(b). Wide binding energy range valence band spectrum in the insert of Fig. 5(a) clearly shows that Eu in $\mathrm{RbEuFe}_{4} \mathrm{As}_{4}$ is in pure $\mathrm{Eu}^{2+}$ state with no contribution from $\mathrm{Eu}^{3+}$ states. It is worth noting that while the resonant data taken with $142 \mathrm{eV}$ photons have a dramatic increase of $\mathrm{Eu} 4 \mathrm{f}$ photoelectron intensity, the inset shows the spectrum obtained with $120 \mathrm{eV}$ with intensity dominating by Fe 3d bands near the Fermi level. Another essential point is that Eu $4 \mathrm{f}$-sensitive ResPES spectra do not show any contribution from the surface $4 \mathrm{f}$ emission, indicating that the analyzed spectral pattern originates from bulk Eu with no presence of the $\mathrm{Eu}^{2+}$ at the surface. The divalent Eu state with a $4 \mathrm{f}^{7}$ electron configuration reveals a large and pure spin magnetic moment $(\mathrm{J}=\mathrm{S}=$ $7 / 2$ ) of $7 \mu_{\mathrm{B}}$, which is responsible for the complex magnetic properties of the Eu sublattice. Note that because the orbital moment of divalent Eu $4 \mathrm{f}$ is equal to zero, this large magnetic moment will be rather insensitive to the crystal-electric field environment. High-resolution ResPES data for $\mathrm{Eu} 4 \mathrm{f}$ states clearly show the $4 \mathrm{f}^{7} \rightarrow 4 \mathrm{f}^{6}$ final-state Eu multiplet where the individual $\mathbf{J}$ components are well resolved [Fig. 5(a)] and in good agreement with both experiment and theoretical calculations $[65,66]$. When following the evolution of the $\mathrm{Eu} 4 \mathrm{f}$ spectral pattern with decreasing temperature [Fig. 5(b)], we can clearly see that there are no apparent changes in its shape and intensity when passing the onset of the superconducting order. However, there is a significant redistribution of the photoemission intensity between the individual J-terms of the $\mathrm{Eu} 4 \mathrm{f}$ multiplet below $14 \mathrm{~K}$. The latter can be explained as the appearance of the long-range FM order of the $4 \mathrm{f}$ moments in the Eu layer $[66,67]$. When the FM order sets in due to exchange interaction, which couples neighboring $4 \mathrm{f}$ moments, the angular momenta assume long-range orientation leading to a preferred direction between incoming light and angular momentum. The latter implies a different excitation probability for the ferromagnetically ordered $4 \mathrm{f}$ states than for the paramagnetically ordered $4 \mathrm{f}$ states with nearly isotropic angular momentum orientation.

Furthermore, in Figs. 5(c) and 5(d) we performed XMCD measurements to directly probe magnetic moments of both $\mathrm{Eu}$ and $\mathrm{Fe}$ atoms. Following standard sum rule analysis, we obtained a large magnetic moment $7.04 \pm 0.01 \mu_{\mathrm{B}}$ for $\mathrm{Eu}$ and a tiny magnetic moment $0.03 \pm 0.01 \mu_{\mathrm{B}}$ for Fe. This value of the local magnetic moment on $\mathrm{Eu}$ is in close agreement with published transport data [15-17]. The DFT-calculated Eu magnetic moment of $6.97 \mu_{\mathrm{B}}$ is in excellent agreement with the experiment and is the same for any considered magnetic configuration.

To investigate magnetic properties of Fe sublattices, we used a disordered local moment (DLM) approach, in which magnetic moments of individual atoms are randomly orientated as in a paramagnetic state [68,69]. This gives the magnitude of Fe magnetic moments of $1.47 \mu_{\mathrm{B}}$. Note, however, that XMCD is sensitive only to the ordered component of the magnetic moment, which is very small, indicating a large fluctuating moment on the Fe sites. FPLO calculations for the experimental structure with the FM order of Eu moments and $\mathrm{U}=5 \mathrm{eV}$ and $\mathrm{J}=1 \mathrm{eV}$ give the Fe moments of $1.7 * 10^{-3} \mu_{\mathrm{B}}$ and $\mathrm{Eu}$ moments of $7.13 \mu_{\mathrm{B}}$. Obtained value of the ordered $\mathrm{Fe}$ moment is consistent with XMCD measurements, while DLM shows the local moment which can be much larger. Applying the magnetic force theorem [70] we found that the exchange interaction between the $\mathrm{Eu}$ and the $\mathrm{Fe}$ moments is rather weak and negative, indicating an AFM coupling between the moments. At the same time, our calculations have shown that AFM90 configuration for Eu moments is the most favorable. The total energy of the AFM180 is of $1.8 \mathrm{meV} / \mathrm{f} . \mathrm{u}$. higher than AFM90, whereas the FM configuration is less favorable among considered being of $4.4 \mathrm{meV} / \mathrm{f}$.u. higher in energy in respect to AFM90.

\section{DISCUSSION}

Using high-resolution ARPES, we observed that the FS of $\mathrm{RbEuFe}_{4} \mathrm{As}_{4}$ consists of multiple holelike and electronlike sheets, similar to other IBSCs. In this unique compound, the itinerant Fe $3 \mathrm{~d}$ electrons at the Fermi level are in proximity to the layers of ferromagnetically ordered large local magnetic moments of $\mathrm{Eu}$ atoms. Therefore, the observed coexistence of $\mathrm{Eu}$ helical AFM order with Fe SC raises a widely discussed question about the possibility of nontrivial superconducting pairing in this material. One of the most direct ways to answer the question about prevailing exotic pairing and its relation to co-existing Eu magnetic order is to probe the symmetry of the superconducting order parameter. $\mathrm{RbEuFe}_{4} \mathrm{As}_{4}$ is an intrinsically hole-doped IBSC with a high transition temperature $\mathrm{T}_{\mathrm{c}} \sim 35 \mathrm{~K}$. Our ARPES data show the opening of a full superconducting gap on all FS sheets below $\mathrm{T}_{\mathrm{c}}$ with an average gap value of $\sim 5 \mathrm{meV}$, in good agreement with momentum-averaged optical conductivity [18], scanning tunneling spectroscopy [44] studies, and our Andreev reflection BJ results. Taking into account similarity of the electronic band structure for $\mathrm{RbEuFe}_{4} \mathrm{As}_{4}$ and $\mathrm{Ba}^{\prime \prime} 122$ " family revealed by ARPES, it is reasonable to speculate that the major contribution into large gap $\Delta_{\mathrm{L}}$ seen in BJ data comes from the electron pockets with the $k_{z}$ independent gap 
and from the inner hole pocket. The only one small gap $\Delta_{\mathrm{S}}$ seen in BJ experiments can be ascribed to the small outer hole pocket superconducting gap with a minor contribution from the strongly $k_{z}$-dependent middle hole pocket gap as in the case of $\mathrm{Ba}_{1-x} \mathrm{~K}_{x} \mathrm{Fe}_{2} \mathrm{As}_{2}$ [55]. With this interpretation, the $\Delta_{\mathrm{S}}$ values derived from $B J$ and ARPES measurements agree with each other.

The fact that the temperature dependence of the various holelike superconducting gaps follows a similar BCS-like relation for different sheets of the FS unambiguously points toward a significant interorbital coupling in this system [71]. The FS topology as well as temperature dependence and anisotropy of the order parameter are very similar for $\mathrm{RbEuFe}_{4} \mathrm{As}_{4}$ and optimally doped $\mathrm{Ba}_{1-x} \mathrm{~K}_{x} \mathrm{Fe}_{2} \mathrm{As}_{2}$. This suggests that $\mathrm{SC}$ in both compounds is of the same origin and is consistent with theoretically proposed $s^{ \pm}$pairing due to the spin fluctuations.

The temperature dependence of the superconducting gaps does not show any uncommon behavior at and below the three-dimensional helical AFM ordering of the $\mathrm{Eu}^{2+}$ spins at $14 \mathrm{~K}$. Moreover, our ARPES data show that weakly dispersing Eu 4f states are at 1-1.7 eV below the Fermi level, and our DFT calculations confirm that these localized states do not hybridize with itinerant $\mathrm{Fe} 3 \mathrm{~d}$ states and therefore do not contribute to the superconducting pairing directly. Both $\mathrm{Fe}-$ and Eu-subsystems are almost decoupled magnetically, since the $\mathrm{Fe} 3 \mathrm{~d}$ bands and the Eu $4 \mathrm{f}$ states are well separated in energy, despite structural proximity of $\mathrm{Fe}-\mathrm{As}$ and Eu layers. Besides, the calculations of the band structure in vicinity of the Fermi level for different types of magnetic orders of Eu moments establish that the topology and the orbital character of $\mathrm{Fe} 3 \mathrm{~d}$ bands are not constrained by the particular magnetic structure. All these observations suggest that the iron-derived states near the Fermi level are completely independent from the localized Eu $4 \mathrm{f}$ electronic subsystem.

Furthermore, we have also addressed the proposition [20] that $\mathrm{SC}$ is responsible for the stabilization of the helical AFM ground state. Using high-resolution resonant photoemission measurements we not only observed the Eu $4 \mathrm{f}$ final state multiplet structure but also, by measuring its temperature and polarization dependence, confirmed in plane ordering of $\mathrm{Eu}^{2+}$ localized moments below $\mathrm{T}_{\mathrm{m}}$. These data also show that the Eu $4 \mathrm{f}^{7} \rightarrow 4 \mathrm{f}^{6}$ final-state multiplet structure and therefore the magnetic arrangement does not change with the onset of SC at $T_{c}$. DFT calculations not only give correct binding energies of the Eu $4 \mathrm{f}$ bands and correct magnetic moments of Eu but also confirm that the helical magnetic structure is the most energetically favorable one.

The tiny magnetic moment of Fe obtained in our XMCD experiment is in agreement with results of Mössbauer spectroscopy measurements [72]. These Mössbauer experiments also suggest that the Fe-subsystem might not be magnetically ordered despite the long-range order of the Eu-subsystem. Using DLM approximation, we show that exchange interaction between $\mathrm{Fe}$ and $\mathrm{Eu}$ is small and negative. Since existing DFT functionals cannot describe correctly charge and spin fluctuations, we cannot determine magnetic order in the $\mathrm{Fe}$ sublattice and assume here that the $\mathrm{Fe}$ moments are disordered. The magnetism of iron in $\mathrm{RbEuFe}_{4} \mathrm{As}_{4}$ could be different from isostructural $\mathrm{CaKFe}_{4} \mathrm{As}_{4}[12,14]$ due to the
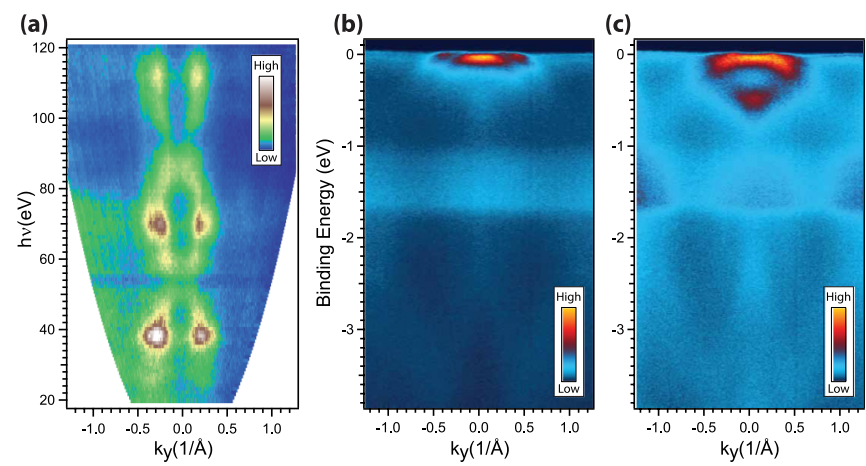

FIG. 6. (a) $k_{y}-k_{z}$ FS map for holelike bands measured with LH photons. (b) Band dispersions in $\mathrm{M}-\Gamma-\mathrm{M}$ direction measured using $113 \mathrm{eV}$ photons and $\mathrm{LH}$ polarization. (c) Band dispersions in A-Z-A direction measured using $89 \mathrm{eV}$ photons and $\mathrm{LH}$ polarization. All data measured at $40 \mathrm{~K}$.

possible biquadratic Eu-Fe coupling [73]. Nevertheless, both magnetic $\mathrm{RbEuFe}_{4} \mathrm{As}_{4}$ and nonmagnetic $\mathrm{CaKFe}_{4} \mathrm{As}_{4}$ compounds have similar FS topology [60]. In both cases, the superconducting gaps for different FS sheets have no clear nodes and are roughly isotropic. These observations once again demonstrate that $\mathrm{SC}$ in $\mathrm{RbEuFe}_{4} \mathrm{As}_{4}$ is completely independent from the magnetic order on Eu. This is in contrast with Eu122 compounds where doping by chemical substitution of Eu sites leads to the suppression of Eu magnetic moments ordering as well as SDW ordering of Fe magnetic moments and enhancement of the superconducting transition temperature $[74,75]$. One should note that in the Eu122 case, doping on Eu-sites inevitably destroys the ordering of the Eu-moments, despite providing charge carriers needed for optimal superconducting pairing. However, one could not imply that SC is strongly coupled to the Eu magnetism in Eu122 compounds because doping on As sites enhances the superconducting pairing with no change to Eu magnetism [76].

$\mathrm{RbEuFe}_{4} \mathrm{As}_{4}$ is one of the few examples of the iron-based superconducting compounds with high transition temperature without additional doping. The SC in this material is enhanced for several reasons: due to the stoichiometric chemical composition, there is no defect scattering detrimental for SC; due to the charge carriers from $\mathrm{Rb}$ layers, it has maximized density of states at the Fermi level beneficial for SC, and it

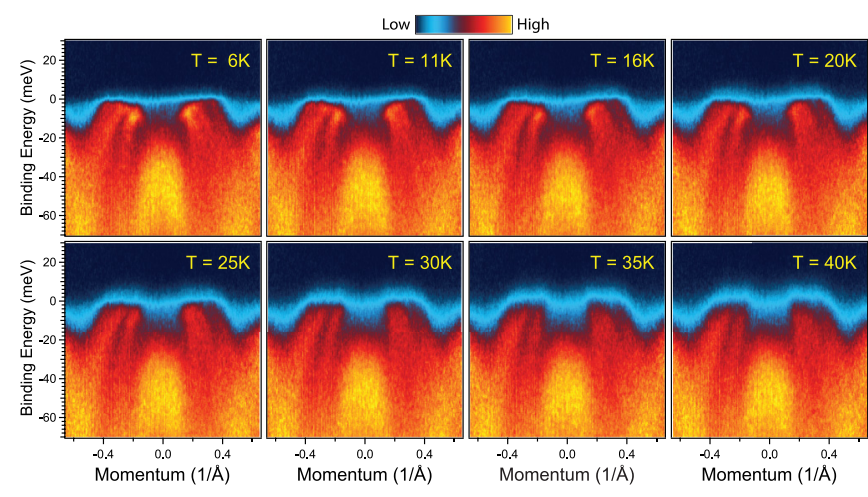

FIG. 7. Band dispersions at Z-point as a function of temperature measured along high symmetry R-Z-R direction. 


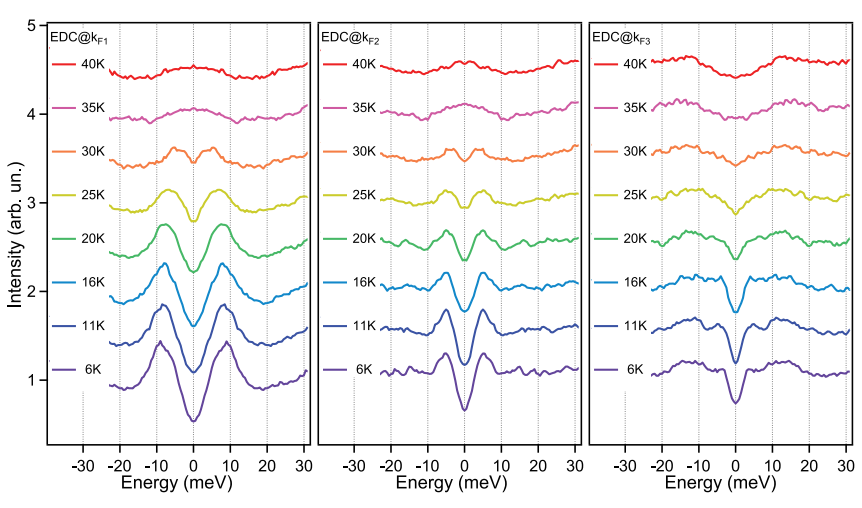

FIG. 8. Temperature dependence of the symmetrised EDCs at equivalent $k_{\mathrm{F}}$ positions for three different FS sheets of the hole pocket at Z-point.

has no long-range magnetic order of iron moments, which is a well-known competitor of the SC. Moreover, a recent study of the doped $\mathrm{RbEu}\left(\mathrm{Fe}_{1-x} \mathrm{Ni}_{x}\right)_{4} \mathrm{As}_{4}$ system shows that by introducing extra electrons with $\mathrm{Ni}$ substitution at doping levels above $x \sim 0.07$, the ferromagnetic superconductor with $\mathrm{T}_{\mathrm{c}}>\mathrm{T}_{\mathrm{m}}$ transforms into a superconducting ferromagnet with $T_{m}>T_{c}$ [77]. This doping-induced interchange opens an unique opportunity to study the underlying change of electronic structure and possible existence of unconventional SC. Therefore, understanding the electronic structure of the parent compound $\mathrm{RbEuFe}_{4} \mathrm{As}_{4}$ becomes extremely important for any future studies of prospective superconducting magnets among iron-based pnictides.

To summarize, we have performed high-resolution ARPES and ResPES studies of a newly discovered IBSC RbEuFe ${ }_{4} \mathrm{As}_{4}$ with helical magnetic order. We observed three holelike FS pockets around $\Gamma$-point and small electronlike pockets around M-point, all formed by Fe-derived bands. Our DFT calculations show that topology and the orbital character of the $\mathrm{Fe} 3 \mathrm{~d}$ band does not strongly depend on the particular magnetic ordering of the Eu $4 \mathrm{f}$ states. A full nodeless superconducting gap with BCS-like temperature dependence has been observed on both hole- and electronlike bands below $\mathrm{T}_{\mathrm{c}}$. In particular, no deviations in the temperature dependence of the order parameter have been detected below the magnetic transition $\mathrm{T}_{\mathrm{m}}$ despite a clear indication for an in-plane magnetic order of $\mathrm{Eu}^{2+}$-localized moments. All these facts unambiguously indicate that Eu-magnetic ordering and the amplitude of the superconducting order parameter (mainly related with Fe electrons) are almost fully decoupled in $\mathrm{RbEuFe}_{4} \mathrm{As}_{4}$.

\section{ACKNOWLEDGMENTS}

We thank Matthew Watson for his critical reading of the manuscript. We thank Diamond Light Source for access to beamline I05 (Proposal No. SI15074 and No. SI19041) that contributed to the results presented here. Work was done using equipment from the LPI Shared Facility Center. K.S.P. and V.M.P. acknowledge support by the Russian Scientific Foundation (RSF Project No. 21-12-00394). A.V.S. and A.S.U. acknowledge support by the Russian Foundation for Basic Research (Project No. 21-52-12043). E.V.C. acknowledges funding by Saint Petersburg State University project for scientific investigations (ID No. 73028629). S.V.E. acknowledges support from the government research assignment for ISPMS SB RAS (Project FWRW-2019-0032). R.V. acknowledges funding by the Deutsche Forschungsgemeinschaft (DFG) TRR 288 (Project A05). V.B. thanks the Goethe University Frankfurt for computational resources and Daniel Guterding for providing the FS plotting software. K.K. thanks M. Valvidares, J. Herrero, H. B. Vasili, S. Agrestini, and N. Brookes for their support during the XMCD experiment at ALBA via IHR Proposal 2019063615. D.V.V. also acknowledges support from the Spanish Ministry of Economy (MAT-2017-88374-P).

\section{APPENDIX A: ARPES}

\section{The $k_{z}$ dependence of the band dispersions}

The $k_{z}$ dependence of the 3D FS measured by varying photon energy is shown in Fig. 6.

\section{Temperature dependence of the superconducting gap for holelike bands}

Temperature dependence of the holelike band dispersions at Z-point $(\mathrm{h} \nu=27 \mathrm{eV})$ is shown in Fig. 7. Symmetrized Energy Distributed Curves (EDCs) at $k_{\mathrm{F}}$ show opening of the superconducting gap for three different FS sheets of the hole pocket at Z-point in Fig. 8. This simplified analysis confirms the results obtained by the fitting to the Dynes function presented in the main text.

\section{APPENDIX B: ANDREEV REFLECTION SPECTROSCOPY}

\section{Temperature dependence of the superconducting gap from "break-junction" spectroscopy}

During sample cleaving in the BJ-experiments, beside the single S-N-S junction, stack contacts are also often formed with sequential chain of the several in-series connected S-N$\mathrm{S}$ junctions junctions $[31,61]$. In this case the stack contact

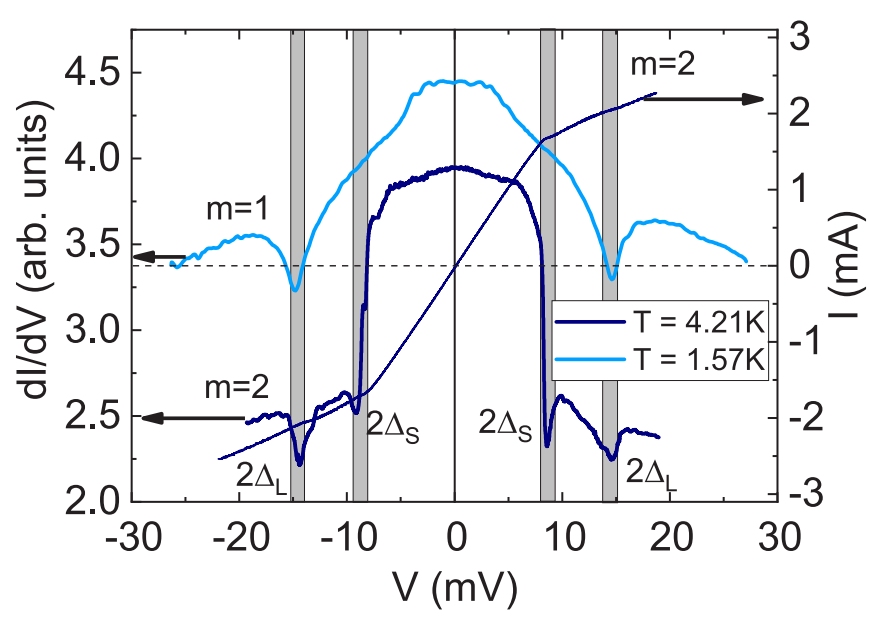

FIG. 9. Typical $I-V$ curve (right Y-axis), and comparison of the dynamic conductance (left Y-axis) for the two different, single- and double- stack junctions. Gray strips highlight the dip positions. 


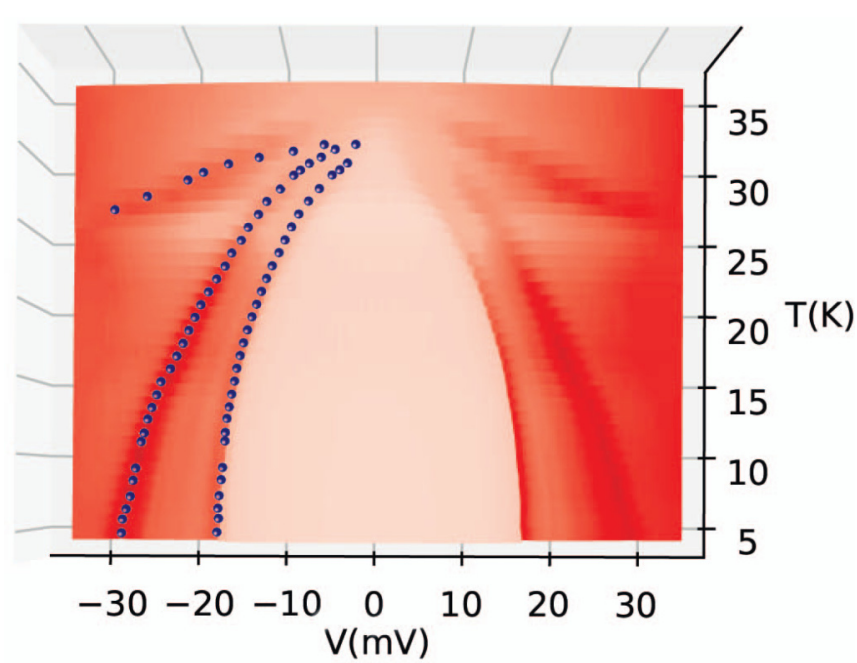

FIG. 10. The 3D-plot of the entire set of $d I / d V$ vs $V$ and $T$ curves for the double-stack junction $m=2$ (the $V_{i}$ values are doubled relative to Fig. 9 .

resonances are observed at bias voltage $V_{m}=m \times\left(2 \Delta_{i} / e\right)$ $(m=2,3, \ldots)$ and may be easily disentangled by scaling down bias voltage with respect to integer $m$.
Particularly, in Fig. 9 we show that the dip positions scale perfectly with $m=1$ for the single-, and $m=2$ junctions, and indicate the presence of two different gaps, the large $\Delta_{\mathrm{L}}$ and the small one $\Delta_{\mathrm{S}}$.

For the double stack contact (Fig. 9) we traced temperature evolution of the spectra in the range $4.2-40 \mathrm{~K}$; the results are shown on Fig. 10. With temperature rising, positions of the dips move to lower bias values and collapse to zero at the same $T_{c}=35 \mathrm{~K}$ which coincides with the bulk value, determined from resistivity measurements and from ARPES.

Beside the two series of minima for two gaps $\Delta_{\mathrm{L}, \mathrm{S}}$ there are additional minima at $\mathrm{T}>30 \mathrm{~K}$. We believe, they are not associated with another gap, otherwise the gap and its $2 \Delta / k_{B} T_{c}$ values would be unphysically large. These dips scale perfectly as the doubled minima of the large gap $(m=2$ junction) and quadruplicated minima of the $m=1$ junction, and have the same temperature dependence. Therefore, we treat this dip as a stack of $m=4$ contacts in series. The $m=4$ junction is pronounced only above $30 \mathrm{~K}$ simply because of insufficient bias voltage for its observation at lower temperatures. Tracing positions of the two major minima we now plot the temperature dependencies of the two gaps on Fig. 3(b).
[1] I. I. Mazin, D. J. Singh, M. D. Johannes, and M. H. Du, Unconventional Superconductivity with a Sign Reversal in the Order Parameter of $\mathrm{LaFeAsO}_{1-x} \mathrm{~F}_{x}$, Phys. Rev. Lett. 101, 057003 (2008).

[2] P. J. Hirschfeld, M. M. Korshunov, and I. I. Mazin, Gap symmetry and structure of Fe-based superconductors, Rep. Prog. Phys. 74, 124508 (2011).

[3] A. Chubukov, Pairing mechanism in Fe-Based superconductors, Annu. Rev. Condens. Matter Phys. 3, 57 (2012).

[4] D. J. Scalapino, A common thread: The pairing interaction for unconventional superconductors, Rev. Mod. Phys. 84, 1383 (2012).

[5] M. Yi, Y. Zhang, Z. X. Shen, and D. Lu, Role of the orbital degree of freedom in iron-based superconductors, npj Quantum Mater. 2, 57 (2017).

[6] P. O. Sprau, A. Kostin, A. Kreisel, A. E. Böhmer, V. Taufour, P. C. Canfield, S. Mukherjee, P. J. Hirschfeld, B. M. Andersen, and J. C. S. Davis, Discovery of orbital-selective Cooper pairing in FeSe, Science 357, 75 (2017).

[7] L. C. Rhodes, M. D. Watson, A. A. Haghighirad, D. V. Evtushinsky, M. Eschrig, and T. K. Kim, Scaling of the superconducting gap with orbital character in FeSe, Phys. Rev. B 98, 180503(R) (2018).

[8] A. V. Chubukov, M. Khodas, and R. M. Fernandes, Magnetism, Superconductivity, Spontaneous Orbital Order in Iron-Based Superconductors: Which Comes First and Why? Phys. Rev. X 6, 041045 (2016).

[9] A. Kreisel, B. M. Andersen, P. O. Sprau, A. Kostin, J. C. Séamus Davis, and P. J. Hirschfeld, Orbital selective pairing and gap structures of iron-based superconductors, Phys. Rev. B 95, 174504 (2017).
[10] A. Iyo, K. Kawashima, T. Kinjo, T. Nishio, S. Ishida, H. Fujihisa, Y. Gotoh, K. Kihou, H. Eisaki, and Y. Yoshida, Newstructure-type Fe-based superconductors: $\mathrm{Ca} A F e_{4} \mathrm{As}_{4}(A=\mathrm{K}$, $\mathrm{Rb}, \mathrm{Cs})$ and $\mathrm{Sr}_{A \mathrm{Fe}_{4}} \mathrm{As}_{4}(A=\mathrm{Rb}, \mathrm{Cs}), \mathrm{J}$. Am. Chem. Soc. 138, 3410 (2016).

[11] K. Kawashima, T. Kinjo, T. Nishio, S. Ishida, H. Fujihisa, Y. Gotoh, K. Kihou, H. Eisaki, Y. Yoshida, and A. Iyo, Superconductivity in Fe-based compound $\mathrm{EuAFe}_{4} \mathrm{As}_{4}(A=\mathrm{Rb}$ and $\mathrm{Cs})$, J. Phys. Soc. Jpn. 85, 064710 (2016).

[12] W. R. Meier, Q. P. Ding, A. Kreyssig, S. L. Bud'ko, A. Sapkota, K. Kothapalli, V. Borisov, R. Valentí, C. D. Batista, P. P. Orth, R. M. Fernandes, A. I. Goldman, Y. Furukawa, A. E. Böhmer, and P. C. Canfield, Hedgehog spin-vortex crystal stabilized in a hole-doped iron-based superconductor, npj Quantum Mater. 3, 5 (2018).

[13] D. E. Jackson, D. VanGennep, W. Bi, D. Zhang, P. Materne, Y. Liu, G-H. Cao, S. T. Weir, Y. K. Vohra, and J. J. Hamlin, Superconducting and magnetic phase diagram of $\mathrm{RbEuFe}_{4} \mathrm{As}_{4}$ and $\mathrm{CsEuFe}_{4} \mathrm{As}_{4}$ at high pressure, Phys. Rev. B 98, 014518 (2018).

[14] V. Borisov, P. C. Canfield, and R. Valentí, Trends in pressureinduced layer-selective half-collapsed tetragonal phases in the iron-based superconductor family $\mathrm{AeAFe}_{4} \mathrm{As}_{4}$, Phys. Rev. B 98, 064104 (2018).

[15] Y. Liu, Y. B. Liu, Z. T. Tang, H. Jiang, Z. C. Wang, A. Ablimit, W. H. Jiao, Q. Tao, C. M. Feng, Z. A. Xu, and G. H. Cao, Superconductivity and ferromagnetism in hole-doped $\mathrm{RbEuFe}_{4} \mathrm{As}_{4}$, Phys. Rev. B 93, 214503 (2016).

[16] J. K. Bao, K. Willa, M. P. Smylie, H. Chen, U. Welp, D. Y. Chung, and M. G. Kanatzidis, Single crystal growth and study of the ferromagnetic superconductor $\mathrm{RbEuFe}_{4} \mathrm{As}_{4}$, Cryst. Growth Des. 18, 3517 (2018). 
[17] M. P. Smylie, K. Willa, J. K. Bao, K. Ryan, Z. Islam, H. Claus, Y. Simsek, Z. Diao, A. Rydh, A. E. Koshelev, W. K. Kwok, D. Y. Chung, M. G. Kanatzidis, and U. Welp, Anisotropic superconductivity and magnetism in single-crystal $\mathrm{RbEuFe}_{4} \mathrm{As}_{4}$, Phys. Rev. B 98, 104503 (2018).

[18] V. S. Stolyarov, A. Casano, M. A. Belyanchikov, A. S. Astrakhantseva, S. Y. Grebenchuk, D. S. Baranov, I. A. Golovchanskiy, I. Voloshenko, E. S. Zhukova, B. P. Gorshunov, A. V. Muratov, V. V. Dremov, L. Y. Vinnikov, D. Roditchev, Y. Liu, G.-H. Cao, M. Dressel, and E. Uykur, Unique interplay between superconducting and ferromagnetic orders in $\mathrm{EuRbFe}_{4} \mathrm{As}_{4}$, Phys. Rev. B 98, 140506(R) (2018).

[19] K. Iida, Y. Nagai, S. Ishida, M. Ishikado, N. Murai, A. D. Christianson, H. Yoshida, Y. Inamura, H. Nakamura, A. Nakao, K. Munakata, D. Kagerbauer, M. Eisterer, K. Kawashima, Y. Yoshida, H. Eisaki, and A. Iyo, Coexisting spin resonance and long-range magnetic order of $\mathrm{Eu}$ in $\mathrm{EuRbFe}_{4} \mathrm{As}_{4}$, Phys. Rev. B 100, 014506 (2019).

[20] Zh. Devizorova and A. Buzdin, Superconductivity-driven helical magnetic structure in $\mathrm{EuRbFe}_{4} \mathrm{As}_{4}$ ferromagnetic superconductor, Phys. Rev. B 100, 104523 (2019).

[21] A. E. Koshelev, Helical structures in layered magnetic superconductors due to indirect exchange interactions mediated by interlayer tunneling, Phys. Rev. B 100, 224503 (2019).

[22] S. Holenstein, B. Fischer, Y. Liu, N. Barbero, G. Simutis, Z. Shermadini, M. Elender, P. K. Biswas, R. Khasanov, A. Amato, T. Shiroka, H. H. Klauss, E. Morenzoni, G. H. Cao, D. Johrendt, and H. Luetkens, Muon spin rotation measurements on $\mathrm{RbEuFe}_{4} \mathrm{As}_{4}$ under pressure (2019), arXiv:1911.04325.

[23] K. Willa, M. P. Smylie, Y. Simsek, J. K. Bao, D. Y. Chung, M. G. Kanatzidis, W. K. Kwok, and U. Welp, Magnetic and superconducting anisotropy in Ni-doped $\mathrm{RbEuFe}_{4} \mathrm{As}_{4}$ single crystals, Phys. Rev. B 101, 064508 (2020).

[24] V. Vlasenko, K. Pervakov, and S. Gavrilkin, Vortex pinning and magnetic phase diagram of EuRbFe $\mathrm{As}_{4}$ iron-based superconductor, Supercond. Sci. Technol. 33, 084009 (2020).

[25] M. Hoesch, T. K. Kim, P. Dudin, H. Wang, S. Scott, P. Harris, S. Patel, M. Matthews, D. Hawkins, S. G. Alcock, T. Richter, J. J. Mudd, M. Basham, L. Pratt, P. Leicester, E. C. Longhi, A. Tamai, and F. Baumberger, A facility for the analysis of the electronic structures of solids and their surfaces by synchrotron radiation photoelectron spectroscopy, Rev. Sci. Instrum. 88, 013106 (2017).

[26] V. Aurich and J. Weule, Non-linear gaussian filters performing edge preserving diffusion, in Mustererkennung 1995, edited by Gerhard Sagerer, Stefan Posch, and Franz Kummert (Springer, Berlin, Heidelberg, 1995), pp. 538-545.

[27] A. Barla, J. Nicolás, D. Cocco, S. M. Valvidares, J. HerreroMartín, P. Gargiani, J. Moldes, C. Ruget, E. Pellegrin, and S. Ferrer, Design and performance of BOREAS, the beamline for resonant X-ray absorption and scattering experiments at the ALBA synchrotron light source, J. Synchrotron Rad. 23, 1507 (2016).

[28] J. Moreland and J. W. Ekin, Electron tunneling experiments using Nb-Sn breakjunctions, J. Appl. Phys. 58, 3888 (1985).

[29] T. E. Kuzmicheva, S. A. Kuzmichev, M. G. Mikheev, Ya. G. Ponomarev, S. N. Tchesnokov, V. M. Pudalov, E. P. Khlybov, and N. D. Zhigadlo, Andreev spectroscopy of iron-based su- perconductors: temperature dependence of the order parameters and scaling of $\Delta_{\mathrm{L}, \mathrm{S}}$ with $T_{\mathrm{C}}$, Phys. Usp. 57, 819 (2014).

[30] R. Kümmel, U. Gunsenheimer, and R. Nicolsky, Andreev scattering of quasiparticle wave packets and current-voltage characteristics of superconducting metallic weak links, Phys. Rev. B 42, 3992 (1990).

[31] T. E. Kuzmicheva, S. A. Kuzmichev, M. G. Mikheev, Ya. G. Ponomarev, S. N. Tchesnokov, Yu. F. Eltsev, V. M. Pudalov, K. S. Pervakov, A. V. Sadakov, A. S. Usoltsev et al., Experimental study of the intrinsic multiple Andreev reflections effect in $\mathrm{GdO}(\mathrm{F}) \mathrm{Fe} A s$ superconductor array junctions, Europhys. Lett. 102, 67006 (2013).

[32] M. Abdel-Hafiez, Y. Zhao, Z. Huang, C.-w. Cho, C. H. Wong, A. Hassen, M. Ohkuma, Y.-W. Fang, B.-J. Pan, Z.-A. Ren, A. Sadakov, A. Usoltsev, V. Pudalov, M. Mito, R. Lortz, C. Krellner, and W. Yang, High-pressure effects on isotropic superconductivity in the iron-free layered pnictide superconductor $\mathrm{BaPd}_{2} \mathrm{As}_{2}$, Phys. Rev. B 97, 134508 (2018).

[33] V. Borisov, S. Biswas, Y. Li, and R. Valentí, Microscopic modeling of correlated systems under pressure: Representative examples, Phys. Status Solidi B 256, 1900229 (2019).

[34] P. E. Blöchl, Projector augmented-wave method, Phys. Rev. B 50, 17953 (1994).

[35] G. Kresse and D. Joubert, From ultrasoft pseudopotentials to the projector augmented-wave method, Phys. Rev. B 59, 1758 (1999).

[36] G. Kresse and J. Hafner, Ab initio molecular dynamics for openshell transition metals, Phys. Rev. B 48, 13115 (1993).

[37] K. Koepernik and H. Eschrig, Full-potential nonorthogonal local-orbital minimum-basis band-structure scheme, Phys. Rev. B 59, 1743 (1999).

[38] M. Geilhufe, S. Achilles, M. A. Köbis, M. Arnold, I. Mertig, W. Hergert, and A. Ernst, Numerical solution of the relativistic single-site scattering problem for the Coulomb and the Mathieu potential, J. Phys.: Condens. Matter 27, 435202 (2015).

[39] J. P. Perdew, K. Burke, and M. Ernzerhof, Generalized Gradient Approximation Made Simple, Phys. Rev. Lett. 77, 3865 (1996).

[40] V. I. Anisimov, J. Zaanen, and O. K. Andersen, Band theory and Mott insulators: Hubbard U instead of Stoner I, Phys. Rev. B 44, 943 (1991).

[41] S. L. Dudarev, G. A. Botton, S. Y. Savrasov, C. J. Humphreys, and A. P. Sutton, Electron-energy-loss spectra and the structural stability of nickel oxide: An LSDA+U study, Phys. Rev. B 57, 1505 (1998).

[42] The binding energy of the Eu 4f states in FPLO changes from -1.3 to $-2.5 \mathrm{eV}$ when the $\mathrm{U}$ parameter is varied between 5 and $7 \mathrm{eV}$, while keeping $\mathrm{J}=1 \mathrm{eV}$.

[43] V. B. Zabolotnyy, D. S. Inosov, D. V. Evtushinsky, A. Koitzsch, A. A. Kordyuk, G. L. Sun, J. T. Park, D. Haug, V. Hinkov, A. V. Boris, C. T. Lin, M. Knupfer, A. N. Yaresko, B. Büchner, A. Varykhalov, R. Follath, and S. V. Borisenko, $(\pi, \pi)$ electronic order in iron arsenide superconductors, Nature (London) 457, 569 (2009).

[44] V. S. Stolyarov, K. S. Pervakov, A. S. Astrakhantseva, I. A. Golovchanskiy, D. V. Vyalikh, T. K. Kim, S. V. Eremeev, V. A. Vlasenko, V. M. Pudalov, A. A. Golubov, E. V. Chulkov, and D. Roditchev, Electronic structures and surface reconstructions in magnetic superconductor EuRbFe ${ }_{4} \mathrm{As}_{4}$, J. Phys. Chem. Lett. 11, 9393 (2020). 
[45] E. van Heumen, J. Vuorinen, K. Koepernik, F. Massee, Y. Huang, M. Shi, J. Klei, J. Goedkoop, M. Lindroos, J. van den Brink, and M. S. Golden, Existence, Character, and Origin of Surface-Related Bands in the High Temperature Iron Pnictide Superconductor $\mathrm{BaFe}_{2-x} \mathrm{Co}_{x} \mathrm{As}_{2}$, Phys. Rev. Lett. 106, 027002 (2011).

[46] N. Mårtensson, B. Reihl, W.-D. Schneider, V. Murgai, L. C. Gupta, and R. D. Parks, Highly resolved surface shifts in a mixed-valent system: $\mathrm{EuPd}_{2} \mathrm{Si}_{2}$, Phys. Rev. B 25, 1446(R) (1982).

[47] W. D Schneider, C. Laubschat, G. Kalkowski, J. Haase, and A. Puschmann, Surface effects in Eu intermetallics: A resonant photoemission study, Phys. Rev. B 28, 2017 (1983).

[48] The relatively large distance between Eu and Fe atoms in this system correspondingly reduced the possibility for hybridization between $\mathrm{Eu}$ and $\mathrm{Fe}$ states.

[49] S. V. Borisenko, D. V. Evtushinsky, Z. H. Liu, I. Morozov, R. Kappenberger, S. Wurmehl, B. Büchner, A. N. Yaresko, T. K. Kim, M. Hoesch, T. Wolf, and N. D. Zhigadlo, Direct observation of spin-orbit coupling in iron-based superconductors, Nat. Phys. 12, 311 (2016).

[50] K. Zantout, S. Backes, and R. Valentí, Effect of Nonlocal Correlations on the Electronic Structure of LiFeAs, Phys. Rev. Lett. 123, 256401 (2019).

[51] S. Bhattacharyya, K. Björnson, K. Zantout, D. Steffensen, L. Fanfarillo, A. Kreisel, R. Valentí, B. M. Andersen, and P. J. Hirschfeld, Non-local correlations in iron pnictides and chalcogenides, Phys. Rev. B 102, 035109 (2020).

[52] A. A. Kordyuk, Electronic band structure of optimal superconductors: From cuprates to ferropnictides and back again (Review Article), Low Temp. Phys. 44, 477 (2018).

[53] H. Ding, P. Richard, K. Nakayama, K. Sugawara, T. Arakane, Y. Sekiba, A. Takayama, S. Souma, T. Sato, T. Takahashi, Z. Wang, X. Dai, Z. Fang, G. F. Chen, J. L. Luo, and N. L. Wang, Observation of Fermi-surface-dependent nodeless superconducting gaps in $\mathrm{Ba}_{0.6} \mathrm{~K}_{0.4} \mathrm{Fe}_{2} \mathrm{As}_{2}$, Europhys. Lett. 83, 47001 (2008).

[54] D. V. Evtushinsky, D. S. Inosov, V. B. Zabolotnyy, A. Koitzsch, M. Knupfer, B. Büchner, M. S. Viazovska, G. L. Sun, V. Hinkov, A. V. Boris, C. T. Lin, B. Keimer, A. Varykhalov, A. A. Kordyuk, and S. V. Borisenko, Momentum dependence of the superconducting gap in $\mathrm{Ba}_{1-x} \mathrm{~K}_{x} \mathrm{Fe}_{2} \mathrm{As}_{2}$, Phys. Rev. B 79, 054517 (2009).

[55] D. V. Evtushinsky, V. B. Zabolotnyy, T. K. Kim, A. A. Kordyuk, A. N. Yaresko, J. Maletz, S. Aswartham, S. Wurmehl, A. V. Boris, D. L. Sun, C. T. Lin, B. Shen, H. H. Wen, A. Varykhalov, R. Follath, B. Büchner, and S. V. Borisenko, Strong electron pairing at the iron $3 \mathrm{~d}_{x z, y z}$ orbitals in hole-doped $\mathrm{BaFe}_{2} \mathrm{As}_{2}$ superconductors revealed by angleresolved photoemission spectroscopy, Phys. Rev. B 89, 064514 (2014).

[56] R. C. Dynes, V. Narayanamurti, and J. P. Garno, Direct Measurement of Quasiparticle-Lifetime Broadening in a StrongCoupled Superconductor, Phys. Rev. Lett. 41, 1509 (1978).

[57] H. Suhl, B. T. Matthias, and L. R. Walker, Bardeen-CooperSchrieffer Theory of Superconductivity in the Case of Overlapping Bands, Phys. Rev. Lett. 3, 552 (1959).

[58] V. A. Moskalenko, The theory of superconductors with overlapping energy bands, Sov. Phys. Usp. 17, 450 (1974).
[59] D. Daghero and R. S. Gonnelli, Probing multiband superconductivity by point-contact spectroscopy, Supercond. Sci. Technol. 23, 043001 (2010).

[60] D. Mou, T. Kong, W. R. Meier, F. Lochner, L.-L. Wang, Q. Lin, Y. Wu, S. L. Bud'ko, I. Eremin, D. D. Johnson, P. C. Canfield, and A. Kaminski, Enhancement of the Superconducting Gap by Nesting in $\mathrm{CaKFe}_{4} \mathrm{As}_{4}$ : A New High Temperature Superconductor, Phys. Rev. Lett. 117, 277001 (2016).

[61] T. E. Kuzmicheva, S. A. Kuzmichev, A. A. Kordyuk, and V. M. Pudalov, Structure and anisotropy of the superconducting order parameter in $\mathrm{Ba}_{0.65} \mathrm{~K}_{0.35} \mathrm{Fe}_{2} \mathrm{As}_{2}$ probed by Andreev spectroscopy, JETP Lett. 107, 42 (2018).

[62] J. Herrero-Martín, V. Scagnoli, C. Mazzoli, Y. Su, R. Mittal, Y. Xiao, T. Brueckel, N. Kumar, S. K. Dhar, A. Thamizhavel, and L. Paolasini, Magnetic structure of $\mathrm{EuFe}_{2} \mathrm{As}_{2}$ as determined by resonant X-ray scattering, Phys. Rev. B 80, 134411 (2009).

[63] Y. Xiao, Y. Su, M. Meven, R. Mittal, C. M. N. Kumar, T. Chatterji, S. Price, J. Persson, N. Kumar, S. K. Dhar, A. Thamizhavel, and Th. Brueckel, Magnetic structure of $\mathrm{EuFe}_{2} \mathrm{As}_{2}$ determined by single-crystal neutron diffraction, Phys. Rev. B 80, 174424 (2009).

[64] In order to increase the contrast, we have used a procedure described in V. Aurich et al. [26].

[65] F. Gerken, Calculated photoemission spectra of the 4f states in the rare-earth metals, J. Phys. F 13, 703 (1983).

[66] K. Starke, Magnetic Dichroism in Core-Level Photoemission (Springer, Berlin, Heidelberg, 2000), p. 136.

[67] K. Starke, E. Navas, E. Arenholz, L. Baumgarten, and G. Kaindl, Circular dichroism in $4 \mathrm{f}$ photoemission from magnetically ordered rare-earth materials, Appl. Phys. A: Mater. Sci. Process. 60, 179 (1995).

[68] B. L. Gyorffy, A. J. Pindor, J. Staunton, G. M. Stocks, and H. Winter, A first-principles theory of ferromagnetic phase transitions in metals, J. Phys. F 15, 1337 (1985).

[69] J. Staunton, B. L. Gyorffy, A. J. Pindor, G. M. Stocks, and H. Winter, Electronic structure of metallic ferromagnets above the Curie temperature, J. Phys. F 15, 1387 (1985).

[70] A. I. Liechtenstein, M. I. Katsnelson, V. P. Antropov, and V. A. Gubanov, Local spin density functional approach to the theory of exchange interactions in ferromagnetic metals and alloys, J. Magn. Magn. Mater. 67, 65 (1987).

[71] To obtain quantitative measure of inter-orbital coupling, one needs data for temperature dependence of the superconducting gaps with higher energy resolution.

[72] M. A. Albedah, F. Nejadsattari, Z. M. Stadnik, Y. Liu, and G. H. Cao, Mössbauer spectroscopy measurements on the $35.5 \mathrm{~K}$ superconductor $\mathrm{Rb}_{1-\delta} \mathrm{EuFe}_{4} \mathrm{As}_{4}$, Phys. Rev. B 97, 144426 (2018).

[73] J. Maiwald, I. I. Mazin, and P. Gegenwart, Microscopic Theory of Magnetic Detwinning in Iron-Based Superconductors with Large-Spin Rare Earths, Phys. Rev. X 8, 011011 (2018).

[74] B. Zhou, L. X. Yang, F. Chen, M. Xu, T. Wu, G. Wu, X. H. Chen, and D. L. Feng, Evolution of electronic structure in $\mathrm{Eu}_{1-x} \mathrm{La}_{x} \mathrm{Fe}_{2} \mathrm{As}_{2}$, J. Phys. Chem. Solids 72, 474 (2011).

[75] Anupam, P. L. Paulose, S. Ramakrishnan, and Z. Hossain, Doping dependent evolution of magnetism and superconductivity in $\mathrm{Eu}_{1-x} \mathrm{~K}_{x} \mathrm{Fe}_{2} \mathrm{As}_{2}(\mathrm{x}=0-1)$ and temperature dependence of the lower critical field $\mathrm{H}_{c 1}$, J. Phys.: Condens. Matter 23, 455702 (2011). 
[76] Y. Tokiwa, S. H. Hübner, O. Beck, H. S. Jeevan, and P. Gegenwart, Unique phase diagram with narrow superconducting dome in $\mathrm{EuFe}_{2}\left(\mathrm{As}_{1-x} \mathrm{P}_{x}\right)_{2}$ due to $\mathrm{Eu}^{2+}$ local magnetic moments, Phys. Rev. B 86, 220505(R) (2012).
[77] Y. Liu, Y. B. Liu, Y. L. Yu, Q. Tao, C. M. Feng, and G. H. $\mathrm{CaO}, \mathrm{RbEu}\left(\mathrm{Fe}_{1-x} \mathrm{Ni}_{x}\right)_{4} \mathrm{As}_{4}$ : From a ferromagnetic superconductor to a superconducting ferromagnet, Phys. Rev. B 96, 224510 (2017). 